\title{
AC 2007-2102: USING FLUID MECHANICS RESEARCH EXAMPLES TO ENHANCE AND STIMULATE UNDERGRADUATE ENGINEERING EDUCATION: PART II
}

\section{Olga Pierrakos, Virginia Tech}

Olga Pierrakos is currently a National Academy of Engineering CASEE AGEP Postdoctoral Engineering Education Researcher (PEER) at Virginia Tech in the Department of Engineering Education. Dr. Pierrakos holds an M.S. in Engineering Mechanics and a Ph.D. in Biomedical Engineering from Virginia Tech. Her Ph.D. work pertained to vortex dynamics in left ventricular flows. She has served as faculty advisor to over thirty mechanical engineering seniors involved in biomedical engineering design projects and taught several mechanical engineering fluid mechanics, design, and technical communication courses. Her research interests are outcomes-based assessment methods for a variety of learning experiences in engineering, students' learning mechanisms, using research and design examples to teach engineering concepts, K-12 engineering education, and cardiovascular fluid mechanics research.

\section{John Charonko, Virginia Tech}

John Charonko is a $\mathrm{PhD}$ student in the School of Biomedical Engineering and Sciences at Virginia Tech. He holds a MS in Engineering Science and Mechanics from Virginia Tech. Currently, his research interests include biomedical applications of fluid mechanics principles, including the study of stent design and how arterial endothelial cells interact with blood flow, and the extension of particle image velocimetry (PIV) techniques to challenging new problems.

\section{Alicia Williams, Virginia Tech}

Alicia Williams is currently pursuing a PhD in Mechanical Engineering at Virginia Tech as a National Science Foundation Graduate Research Fellow. Her research interests beyond engineering education include laminar mixing techniques and novel drug delivery systems using ferrofluid and magnetic fields.

\section{Satyaprakash Karri, Virginia Tech}

Satya prakash Karri is currently a PhD student in the School of Biomedical Engineering and Sciences at Virginia Tech. Karri holds a M.S in Mechanical Engineering from UT Arlington. His research interests are in bio-fluid mechanics, turbulence, FEA, CFD and composite structures.

\section{Kelley Stewart, Virginia Tech}

Kelley Stewart is currently pursuing her Master of Science degree in Mechanical Engineering at Virginia Tech. Her current research interests include left ventricle vortex dynamics under diseased conditions, arterial flows, and engineering education.

\section{Pavlos Vlachos, Virginia Tech}

Dr Vlachos is assistant professor in the Mechanical Engineering Dept at Virginia Tech. He received his BS in Mechanical Engineering from the National Technical University of Athens (1995) and his MS (1998) and PhD (2000) in Engineering Mechanics from Virginia Tech. His research focuses on experimental fluid mechanics addressing a variety of flows, primarily, wall bounded flows, vortex dynamics, biofluid mechanics and multi-phase flows as well as engineering education. 


\section{Using Fluid Mechanics Research Examples to Enhance and Stimulate Undergraduate Engineering Education}

\section{Introduction}

Approximately $62 \%$ of the undergraduate students who graduated in 2000 with an engineering B.S. in the United States received their degree from Research I and II institutions. ${ }^{1}$ Although these universities successfully recruit their undergraduates by proudly displaying their research infrastructure and state-of-the-art facilities, a vast majority of these students graduate without ever being exposed to these assets. Even those students who are introduced to research often remain oblivious to the rich research diversity and the multi-disciplinary culture of engineering. This is an increasingly important concern because the future engineer is expected to adapt to a varying and continuously evolving environment while simultaneously being able to operate outside the narrow limits of one discipline, crossing over boundaries and interfacing between different fields. In recent years, the Boyer Commission, ${ }^{1}$ the National Science Foundation, ${ }^{2}$ the American Association for the Advancement of Science, ${ }^{3}$ and the National Research Council ${ }^{4}$ have urged universities to make "research-based learning the standard" for undergraduate education. Participation in research deepens a student's understanding and promotes the communication and teamwork needed to solve complex problems. Enabling students to be part of the intellectual process and instills in them a sense of fulfillment and imparts life-long benefits. A report, released on June 2005 by the National Academy of Engineering, further supports these arguments. ${ }^{5}$ The report considered current engineering education, inadequate to prepare future engineers and suggested that BS graduates should be considered engineers in training and an MS should be a professional degree. This finding illustrates the need at the undergraduate level for "research-based learning" which is inherent in the graduate level but almost non-existent in the undergraduate level.

To achieve this research-based learning at the undergraduate level, a new educational paradigm is needed that, demands a commitment to the intellectual growth of individual students, redefines the role of engineering in society, and stimulates students to pursue careers in engineering and research. These goals can be accomplished by integrating research into engineering education, serving to increase recruitment and retention and enabling future engineers to become society leaders.

To pursue these goals, we initiated an effort to translate state-of-the-art multidisciplinary research examples and accomplishments to the classroom. More specifically, in our previous conference paper to ASEE last year, we presented the development of a research transfer model for translating state-of-the-art fluid mechanics and biofluids research into the engineering education of students from the high school level to freshmen engineers. The model was implemented through a series of presentations and hands-on exercises. This previous effort showed much promise as a model for transferring engineering research to the high school and freshmen levels. 
By applying the lessons we learned, our current goal is to expand this research transfer to a larger pool of engineering students at the varying academic levels. The five main questions guiding this effort were:

1) What are the learning outcomes for these students during this experience?

2) Did this intervention aid in recruiting and retaining engineering students?

3) Did this intervention influence the engineering students to apply and get involved in undergraduate research?

4) Has this intervention influenced the career path of the students (i.e. graduate school or other research position)?

5) Is the intervention more effective at specific academic level(s)?

In this effort, we have placed particular emphasis on transferring research to groups under-represented in engineering and encouraging the students to engage in hands-on research. The progression of research transfer through the different levels of engineering education is illustrated in Figure 1. At the end of this development ladder, we find the future interdisciplinary engineers who are leaders in industry, technology, and academia. In this effort, via research transfer and examples, another goal is the recruitment of middle school and high school students and the retention of freshman engineers. Recruiting and retention can be increased by creating awareness and improving the image and perceptions of engineering during the early educational stages. This goal will be accomplished by navigating the students through the maze of engineering fields using as "icons" visual and experiential stimulations adopted from everyday examples that are related to observations in nature or research applications.

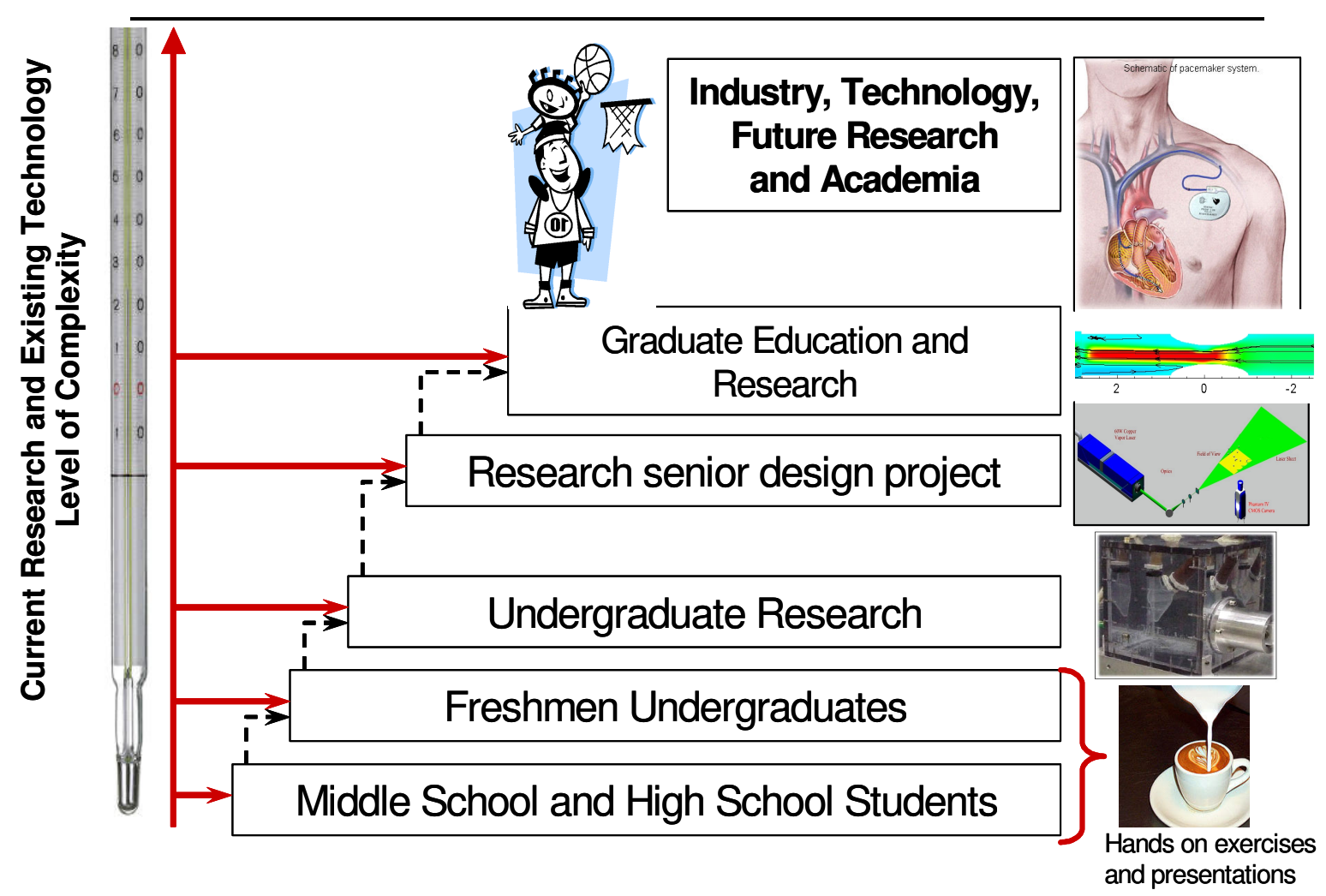

Figure 1. Schematic of the development ladder of research translation to engineering education from middle school to graduate education. This illustration shows the big picture of research transfer leading to interdisciplinary engineers who are leaders in industry, technology, and academia. 
The assessment of the research transfer intervention is implemented by use of pre- and post-surveys. The population of students included freshmen engineering students, sophomore and junior mechanical engineers, and engineering graduate students at Virginia Tech in the Department of Mechanical Engineering. Over 450 students participated in this effort within one semester. Lastly, this research transfer model and assessment instrument can be useful to other engineering disciplines.

\section{Background: What We Mean by Research Transfer}

This paper presents the transfer of recent interdisciplinary engineering research in fluid mechanics and cardiovascular mechanics from the freshman to the graduate classroom in order to meet the following specific aims:

Specific Aim 1: Give students the opportunity to explore the diversity of engineering fields by using tangible and intuitive examples and integrating them with contemporary research applications.

Specific Aim 2: Demonstrate how seemingly diverse areas of research are connected through the same fundamental engineering principles and how these very same principles apply and govern our everyday reality.

Specific Aim 3: Inspire the students to pursue a career in engineering and research, thus supporting student recruitment into engineering (for undecided undergraduates) or into graduate school (for undergraduates). This aim also supports retention.

Our expectations are that our research transfer will have the following effects on the students: (1) the student's intuition should be sharpened, and (2) the student's perception about engineering should be broadened. By improving the students' ability to experience and interpret his or her physical environment, the undergraduate engineers will be stimulated to engage in undergraduate research and potentially transition towards graduate studies. The research was transferred through a series of presentations and hands-on exercises delivered to students from the freshman to the graduate level with these backgrounds:

1) Freshman Engineering Students: Students participating in learning communities in ongoing programs sponsored by the Center for the Enhancement for Engineering Diversity (CEED) at Virginia Tech. Hypatia, a learning community for first-year women engineering students, and Galileo, a learning community for men in engineering, were the two freshman student groups. These learning communities are designed to bring together students in a residential environment to provide encouragement and support in their pursuit of a career in engineering.

2) Sophomore Engineering Students: Students in mechanical engineering taking the sophomore level thermal-fluids engineering course. This is the first course in mechanical engineering that introduces the students to thermodynamics, fluid mechanics, and heat transfer. 
3) Junior Engineering Students: Students in mechanical engineering taking a junior level fluid mechanics course. This is the fluid mechanics course succeeding the sophomore level thermalfluids course.

4) Graduate Engineering Students: Students taking a fundamentals fluid dynamics graduate level course in the mechanical engineering department. The graduate students taking this course, $60 \%$ of whom are involved with graduate-level fluid mechanics research, were from multiple engineering departments.

Of the approximately 480 ( $25 \%$ of whom were female) students from the four groups exposed to the fluid mechanics research transfer intervention this past semester (Fall 2006), 380 of these students participated in taking the pre- and post-survey instruments. The assessment results in this paper correspond to these students. Table 1 illustrates the student population in terms of gender and race.

Table 1: Student population for the four student groups based on gender and race.

\begin{tabular}{|l|c|c|c|c|}
\hline $\begin{array}{c}\text { Student Population } \\
\text { (Gender and Race) }\end{array}$ & $\begin{array}{c}\text { Freshman } \\
\text { Students }\end{array}$ & $\begin{array}{c}\text { Sophomore } \\
\text { Students }\end{array}$ & $\begin{array}{c}\text { Junior } \\
\text { Students }\end{array}$ & $\begin{array}{c}\text { Graduate } \\
\text { Students }\end{array}$ \\
\hline Number of Survey Participants (380) & 250 & 48 & 68 & 14 \\
\hline Female & $30 \%$ & $10 \%$ & $5 \%$ & $25 \%$ \\
Male & $70 \%$ & $90 \%$ & $95 \%$ & $75 \%$ \\
African American & $5 \%$ & $5 \%$ & $2 \%$ & $8 \%$ \\
Asian & $6 \%$ & $8 \%$ & $4 \%$ & $8 \%$ \\
Caucasian & $82 \%$ & $82 \%$ & $87 \%$ & $75 \%$ \\
Hispanic & $2 \%$ & $3 \%$ & $2 \%$ & $9 \%$ \\
Other & $5 \%$ & $2 \%$ & $5 \%$ & $0 \%$ \\
\hline
\end{tabular}

\section{Methods}

The transfer of the research was implemented in three steps: pre-assessment, presentation and demonstrations, post-assessment. The pre- and post-assessments are discussed in more detail in the following section. The seminar-style presentation, which was approximately 60-minutes and was designed to meet Bloom's taxonomy of low-level learning objectives, was given to the four student groups and designed so as to be easily adapted to the dynamics and knowledge base of each group, while demonstrating how the same basic principles apply to different fields. The presentation, demonstrations, and exercises were designed to do the following: (1) to explore the diversity of fluids and biomedical engineering fields by using tangible and intuitive real life examples and integrating them with contemporary research; and (2) to demonstrate how seemingly diverse areas of research are connected through fundamental engineering principles and how these principles occur in everyday physical or technological processes. One comparison the authors attempted to illustrate during the interventions is shown in Figure 2. This slide shows a left-ventricular assist device used to support patients awaiting heart transplantation and a typical jet engine. The grouping of these two examples illustrates how the concept of a fan/compressor is as applicable to aeronautics as it is to biomedical engineering. By exploring a variety of engineering applications and their impact to society, student's perception of 
engineering is improved and the strong effects that engineering research and technology have on our everyday life are illustrated.

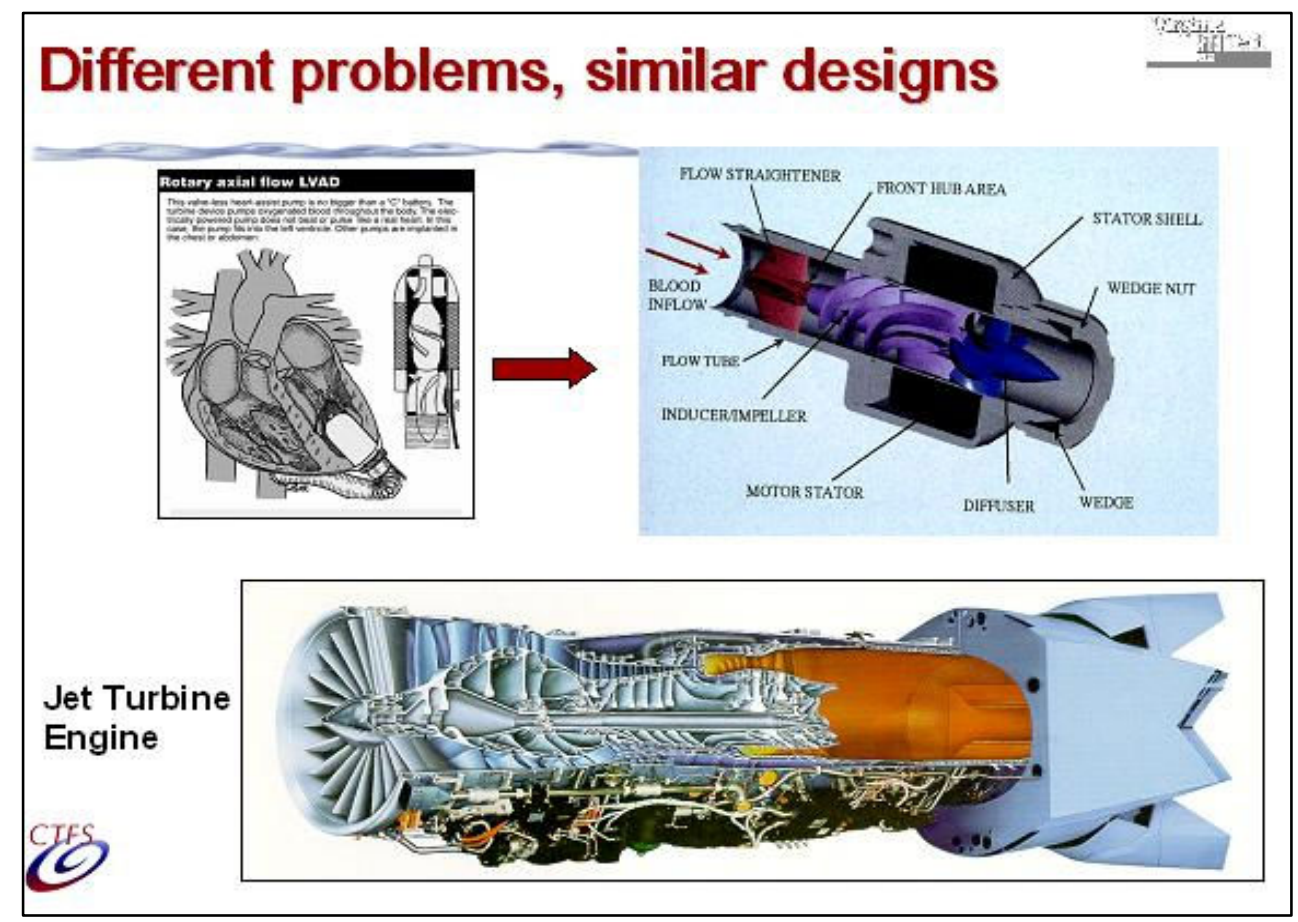

Figure 2. A slide from the intervention showing similar engineering principles between the design of the left ventricular assist device (LVAD) and turbine engine.

Another part of the intervention attempted to appeal to the students' knowledge through their everyday experiences while developing their intuitive understanding of fluids physics. Most people have witnessed or participated in sports to some degree, and are familiar with the curve balls thrown by baseball and softball pitchers. Another similar example is the "banana kick" in soccer that has a curved trajectory and is often used to deceive goaltenders. However, the fluid physics behind these phenomena are often neglected. Both of these particular phenomena are associated with the Magnus effect and Bernoulli's principle, which makes use of additional fluid entrainment based on the rotation of the ball to generate forces perpendicular to the spin and velocity axes. An additional example from sports that is associated with fluids is the dimpling of golf balls to reduce drag in the turbulent flows in which they operate. These examples and their physics are shown in Figure 3. Although we hope to have the details of the intervention and the presentation slides available online for a wider distribution in the future, at this time this is not currently available. 


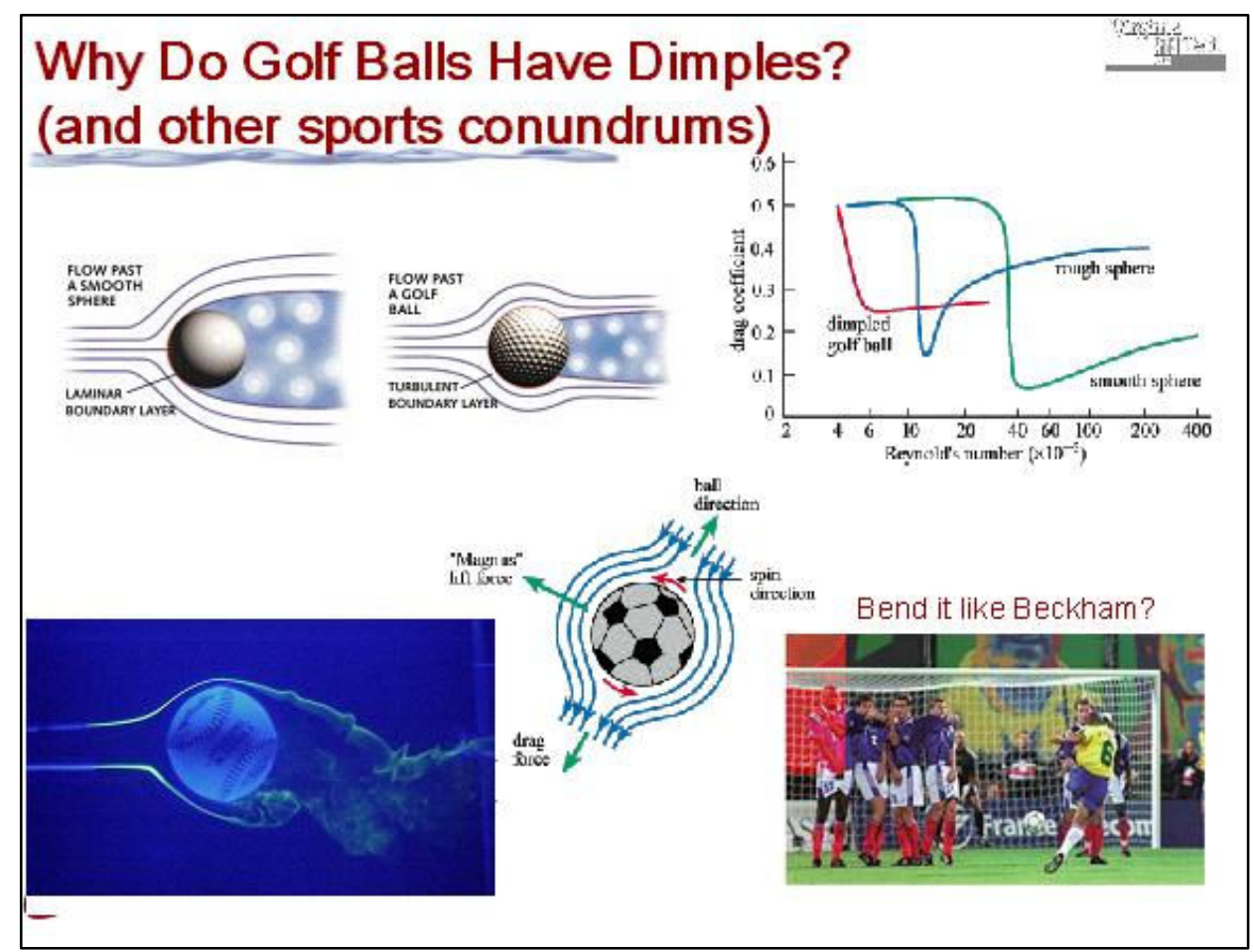

Figure 3. Slide from the interventions showing fluids-related examples from sports.

The specific objectives varied slightly for each group of students taking into consideration each group's educational level. For the freshmen engineers, the main objective was to introduce them to various research areas as a means of illustrating the diversity of research and the overlap of fundamental engineering concepts. For the sophomore and junior engineers, the objectives also included more scientific and technical discussion of these research examples to encourage the application of their coursework to actual engineering problems. Finally, for the graduate students, an additional objective was to deepen their fundamental knowledge and challenge them to apply their previous coursework to the examples shown and their own research. The level of complexity and detail of engineering concepts within the presentation was also tailored to the academic level each student group. However, the slides themselves remained nearly unchanged between presentations, the main difference was the level of discussion and questioning during each presentation.

Upon completion of the presentation and demonstration, our objectives were for the students to be able to:

1. articulate and recognize the role and importance of engineering in society,

2. explore the diversity of fluid mechanics and biomedical engineering fields by using tangible and intuitive real life examples,

3. understand how seemingly diverse areas of research are connected through fundamental engineering principles and how these principles occur in everyday physical or technological processes,

4. appreciate the interdisciplinary and multidisciplinary character of modern engineering,

5. develop an awareness of emerging engineering fields and of future research trends and challenges. 
Lastly, we assessed how well these interventions addressed ABET's "3a through k" criteria which state that: "engineering programs must demonstrate that their graduates have:

(a) an ability to apply knowledge of mathematics, science, and engineering,

(b) an ability to design and conduct experiments, as well as to analyze and interpret data,

(c) an ability to design a system, component, or process to meet desired needs,

(d) an ability to function on multidisciplinary teams,

(e) an ability to identify, formulate, and solve engineering problems,

(f) an understanding of professional and ethical responsibility,

(g) an ability to communicate effectively,

(h) the broad education necessary to understand the impact of engineering solutions in a global and societal context,

(i) a recognition of the need for, and ability to engage in, lifelong learning,

(j) a knowledge of contemporary issues;

(k) an ability to use the techniques, skills, and modern engineering tools necessary for engineering practice."

Six of the eleven criteria are in bold because we feel that there were several research examples discussed during the presentation that specifically addressed these six ABET criteria. For example, in addressing the impact of engineering solutions in a global and societal setting as well as ethical responsibility, the Tacoma-Narrows Bridge (which collapsed in 1941 due to a failure to consider the interaction of the bridge's fluid mechanics with its structural mechanics) example was discussed in depth. Another example was discussed at length in each intervention was the Citicorp building in New York city (which was repaired after being constructed due to a fluid and structural mechanics-related design flaw). This particular example also highlighted ethical issues in engineering, as it involved issues of accountability in engineering design.

Each intervention was presented by two graduate students studying a variety of fluid mechanics problems for their master's or $\mathrm{PhD}$ research. The presenters of these interventions also benefited from them and the presentations served as a valuable teaching experience in that:

1. They were required to consider the students' perspectives on research and technical fluid mechanics in preparing for the presentations.

2. They learned to interweave variety of examples from multiple disciplines with proper transitions to exemplify the importance of fluid mechanics and to bring about a unified theme.

3. They learned a suggestive methodology in their presentations to bring about participation from the students and to enhance their learning.

4. They were required to think about the presentations as a vehicle to transfer the idea of fluid mechanics principles being applied across various disciplines, rather than just as a medium for presentation of fluid mechanics facts and theories

5. It also promoted their ability to work in a team, bringing their ideas together

6. It nurtured in them the importance of Research Transfer in effective engineering instruction.

In doing so, the important skill of explaining difficult concepts to a variety of educational and personal backgrounds was fostered. 


\section{Assessment Results and Discussion}

The assessment of the research transfer was done by use of pre- and post-surveys, which compared the student's perceptions of engineering, research, fluid mechanics, and discipline diversity. The pre-assessment involved a component to establish the student's knowledge base and background, while the post-assessment included additional feedback and an overall evaluation of the experience. Most of the survey questions were based on a Likert scale of 1 (strongly disagree) to 5 (strongly agree). The pre- and post-surveys were approved by the Institutional Review Board (IRB) of the Office of Research Compliance.

\section{Students' Assessment of Engineering and Research}

In the pre- and post- surveys, students were asked several questions pertaining to knowledge of engineering diversity and research. Table 2 shows the results of four survey questions for the freshman, sophomore, and junior student groups. For each group, pre- and postsurvey mean ratings are given as well as the percentage of students that "agreed" and "strongly agreed" with the statement.

To better educate the students that engineers transcend the boundaries of a singlediscipline, during the 60-minute research transfer presentation given to the students, several examples were given to illustrate the multidisciplinarity of most engineering projects in industry and academic settings. In order to assess the impact of this type of research examples, students were asked "which of the following engineering disciplines are involved in the entire infrastructure of designing an automobile?" Since this example was not discussed in particular during the presentation, though a similar examples concerning a fighter jet and desktop computers were investigated, students were required to transfer the concepts illustrated in the presentation to another field. From a list of eight engineering disciplines the students could select from, they acknowledged that mechanical, electrical, computer, and industrial systems engineers were involved. This is a finding that we expected. The statistically significant differences existed for the following engineering disciplines: civil engineers, biomedical engineers, and aerospace engineers. So, in being exposed to research examples pertinent to this question during the presentation: (a) 31\%-49\% more students agreed that the civil engineers were involved, (b) 18\%-23\% more students agreed that the aerospace engineers were involved, and (c) 34\%-50\% more students agreed that the biomedical engineers were involved. This was a useful observation that illustrated that by exposing students to research examples, the multidisciplinary and interdisciplinary nature of engineering can be taught.

Statistically significant differences, between the pre- and post- survey ratings, were also observed when the students were asked if "research has a large impact on my daily life" and if "research is as important to engineering as it is to science." There were 15\%, 20\%, and 17\% more students, respectively for the freshmen, sophomores, and juniors, that either "agreed" or "strongly agreed with the impact research has on their life. When asked about research being as important to engineering as it is to science, overall students knew this was true and the percent difference overall were not significant. What was significant was that after the presentation, $19 \%$ and $12 \%$ more sophomore and junior students "strongly agreed" with the statement. Interestingly, the trend was opposite among the freshman, with similar total agreement before 
and after, but a 5 percentage point shift from "strongly agree" back to "agree." This change was not statistically significant, however.

The last of the research questions was about "considering doing undergraduate research in the future." $68 \%$ of the freshman and sophomore students either "agreed" or "strongly agreed" that they would consider doing undergraduate research. This corresponded to a 7\% and $8 \%$ difference, respectively for the two groups, when comparing pre- and post- results. As for the juniors, $10 \%$ of them that originally "agreed" changed their answer to "strongly agree." This is a important outcome for the students because it appears that they highly valued the presentation as well as learned a lot from it.

Table 2: Student survey questions and results pertaining to knowledge of engineering diversity and research. For each of the student groups, mean ratings and percentage of students that "agreed" and "strongly agreed" with the statement are included. Statistically significant differences were found for the statements with an asterisk [*p $<0.05$ and $* * p<0.01]$.

\begin{tabular}{|c|c|c|c|c|c|c|c|c|c|c|}
\hline \multirow{2}{*}{\multicolumn{2}{|c|}{$\begin{array}{c}\text { Questions about } \\
\text { Engineering and Research }\end{array}$}} & \multicolumn{3}{|c|}{ Freshmen } & \multicolumn{3}{|c|}{ Sophomores } & \multicolumn{3}{|c|}{ Juniors } \\
\hline & & Pre & Post & Diff & Pre & Post & Diff & Pre & Post & Diff \\
\hline \multicolumn{11}{|c|}{$\begin{array}{l}\text { Which of the following engineering disciplines are involved in the entire infrastructure of } \\
\text { designing an automobile? Please select all that apply. } * p<0.05 \text { and } * * p<0.01\end{array}$} \\
\hline \multirow{8}{*}{1} & Mechanical engineers & $98 \%$ & $98 \%$ & $0 \%$ & $100 \%$ & $100 \%$ & $0 \%$ & $99 \%$ & $100 \%$ & $1 \%$ \\
\hline & Materials engineers & $93 \%$ & $97 \%$ & $4 \%$ & $93 \%$ & $100 \%$ & $7 \%$ & $97 \%$ & $97 \%$ & $0 \%$ \\
\hline & Civil engineers & $40 \%$ & $72 \%$ & $31 \% * *$ & $25 \%$ & $74 \%$ & $49 \% * *$ & $32 \%$ & $64 \%$ & $32 \% * *$ \\
\hline & Aerospace engineers & $58 \%$ & $77 \%$ & $19 \% * *$ & $54 \%$ & $72 \%$ & $18 \% * *$ & $58 \%$ & $81 \%$ & $23 \% * *$ \\
\hline & Biomedical engineers & $19 \%$ & $53 \%$ & $34 \% * *$ & $29 \%$ & $79 \%$ & $50 \% * *$ & $28 \%$ & $66 \%$ & $38 \% * *$ \\
\hline & Industrial systems engineers & $77 \%$ & $88 \%$ & $11 \% *$ & $82 \%$ & $95 \%$ & $13 \% *$ & $81 \%$ & $92 \%$ & $12 \% *$ \\
\hline & Computer engineers & $82 \%$ & $92 \%$ & $10 \% *$ & $86 \%$ & $100 \%$ & $14 \% *$ & $90 \%$ & $95 \%$ & $5 \%$ \\
\hline & Electrical engineers & $94 \%$ & $97 \%$ & $3 \%$ & $100 \%$ & $100 \%$ & $0 \%$ & $94 \%$ & $97 \%$ & $2 \%$ \\
\hline
\end{tabular}

Research has a large impact on my daily life. $* * p<0.01$

\begin{tabular}{|l|c|c|c|c|c|c|c|c|c|}
\hline Mean Rating & $\mathbf{3 . 7 9}$ & $\mathbf{4 . 1 2}$ & $\mathbf{8 \%} * *$ & $\mathbf{3 . 7 9}$ & $\mathbf{4 . 3 3}$ & $\mathbf{1 3 \%} * *$ & $\mathbf{3 . 6 0}$ & $\mathbf{4 . 1 1}$ & $\mathbf{1 2 \%} * *$ \\
\cline { 2 - 10 } & $43 \%$ & $45 \%$ & $2 \% * *$ & $34 \%$ & $28 \%$ & $-6 \% * *$ & $46 \%$ & $41 \%$ & $-5 \% * *$ \\
\hline Strongly Agree & $23 \%$ & $36 \%$ & $13 \% * *$ & $28 \%$ & $54 \%$ & $26 \% * *$ & $17 \%$ & $39 \%$ & $22 \% * *$ \\
\hline Agree and Strongly Agree & $66 \%$ & $81 \%$ & $15 \% * *$ & $62 \%$ & $82 \%$ & $20 \% * *$ & $63 \%$ & $80 \%$ & $17 \% * *$ \\
\hline
\end{tabular}

Research is as important to engineering as it is to science. ** $\mathbf{p}<0.01$

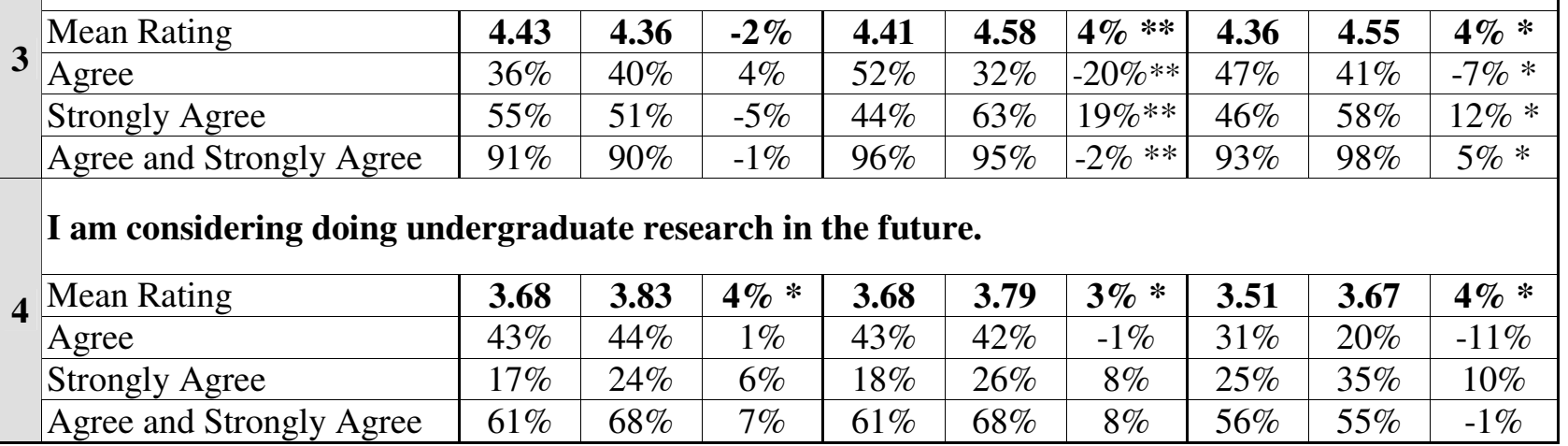




\section{Students' Assessment of Fluid Mechanics}

Considering that the majority of the presentation pertained to fluid mechanics research examples, in the pre- and post- surveys, students were also asked several questions regarding fluid mechanics. Table 3 shows the results of five such survey questions for the freshman, sophomore, and junior student groups. For each group, pre- and post-survey mean ratings are given as well as the percentage of students that "agreed" and "strongly agreed" with the statement. Two of these questions, in particular, that revealed statistically significant differences, between the pre- and post- survey ratings, were "fluid mechanics is a fun and exciting field of study" and "fluid mechanics has a large impact on my daily life." As it turns out, after the presentation, 31\% ( $\mathrm{p}<0.01)$ more sophomores and 15\% $(\mathrm{p}<0.01)$ more juniors either "agreed" or "strongly agreed" with the statement that fluid mechanics is an exciting field. These differences are even more pronounced as well as statistically significant in the second statement, $24 \%$ to $34 \%$ ( $\mathrm{p}<0.01$ ) more students (answering "agreed" or "strongly agreed") recognized that fluid mechanics has a large impact on their daily life. This was an important outcome not only because so many students recognized the impact of fluid mechanics, but also because students at all levels acknowledged this effect. Even the graduate students, most of whom are doing fluid mechanics research at the graduate-level, improved in their perception of the influence and relevance of fluid mechanics in their lives.

The next two statements students were asked in the pre- and post- surveys pertained to "a good understanding of fluid mechanics is essential for most engineering disciplines/departments" and "fluid mechanics research examples should be presented in the classroom." For both of these statements, although the higher percent differences (between pre and post ratings) were observed for the younger students, it was the graduate students and juniors that had the higher ratings, as we would expect. Lastly, the last of the fluid mechanics questions was "I am interested in getting involved with fluid mechanics related research," which the sophomores "strongly agreed" with by having an $11 \%$ difference $(\mathrm{p}<0.05)$, and "agreed" with a $15 \%$ difference $(p<0.01)$. The juniors did not have this kind of response and the reason for that could be that they feel they are too close to the end of their degree and not enough time is left for them to get involved with research. Or maybe they have already decided on their field of expertise (i.e. robotics, solid mechanics, controls, etc.), which is not fluid mechanics. 
Table 3: Student survey questions and results pertaining to knowledge and value of fluid mechanics. For each of the student groups, mean ratings and percentage of students that "agreed" and "strongly agreed" with the statement are included. Statistically significant differences were found for the statements with an asterisk $[* \mathrm{p}<0.05$ and $* * \mathrm{p}<0.01]$.

\begin{tabular}{|c|c|c|c|c|c|c|c|c|c|c|c|c|c|}
\hline & \multirow{2}{*}{$\begin{array}{l}\text { Questions about } \\
\text { Fluid Mechanics }\end{array}$} & \multicolumn{3}{|c|}{ Freshmen } & \multicolumn{3}{|c|}{ Sophomores } & \multicolumn{3}{|c|}{ Juniors } & \multicolumn{3}{|c|}{ Grad Students } \\
\hline & & Pre & Post & Diff & Pre & Pre & Pre & Pre & Post & Diff & Pre & Post & Diff \\
\hline \multirow{5}{*}{1} & \multicolumn{13}{|c|}{ Fluid mechanics is a fun and exciting field of study. } \\
\hline & Mean Rating & NA & NA & NA & 3.11 & 3.74 & $17 \% * *$ & 3.60 & 3.89 & $8 \% * *$ & NA & NA & NA \\
\hline & Agree & NA & NA & NA & $29 \%$ & $47 \%$ & $19 \%$ & $51 \%$ & $56 \%$ & $5 \%$ & NA & NA & NA \\
\hline & Strongly Agree & NA & NA & NA & $4 \%$ & $16 \%$ & $12 \%$ & $7 \%$ & $17 \%$ & $10 \%$ & NA & NA & NA \\
\hline & Agree and Stron & NA & NA & NA & $32 \%$ & $63 \%$ & $31 \%$ & $58 \%$ & $73 \%$ & $15 \%$ & NA & NA & NA \\
\hline \multirow{5}{*}{2} & \multicolumn{13}{|c|}{ Fluid mechanics has a large impact on my daily life. } \\
\hline & & 3.46 & 4.16 & $17 \%$ ** & 3.70 & 4.31 & $14 \% * *$ & $\mathbf{3 . 7 5}$ & 4.28 & $12 \% * *$ & 4.00 & 4.31 & $7 \% *$ \\
\hline & & $37 \%$ & $43 \%$ & $6 \%$ & $53 \%$ & $62 \%$ & $8 \%$ & $53 \%$ & $56 \%$ & $3 \%$ & $31 \%$ & $6 \%$ & $15 \%$ \\
\hline & & $12 \%$ & $39 \%$ & $27 \%$ & $11 \%$ & $36 \%$ & $25 \%$ & $14 \%$ & $36 \%$ & $22 \%$ & $38 \%$ & $46 \%$ & $9 \%$ \\
\hline & & $49 \%$ & $82 \%$ & $33 \%$ & $64 \%$ & $97 \%$ & $34 \%$ & $67 \%$ & $92 \%$ & $26 \%$ & $69 \%$ & $92 \%$ & $24 \%$ \\
\hline & \multicolumn{13}{|c|}{ A good understanding of fluid mechanics is essential for most engineering disciplines/departments. } \\
\hline \multirow{4}{*}{3} & Mean Rating & 3.46 & 3.89 & $11 \% *$ & 3.87 & 4.15 & $7 \% *$ & 3.86 & 4.11 & $6 \% *$ & 4.19 & 4.08 & $-3 \%$ \\
\hline & Agree & $42 \%$ & $47 \%$ & $6 \%$ & $64 \%$ & $58 \%$ & $-6 \%$ & $71 \%$ & $59 \%$ & $-11 \%$ & $56 \%$ & $46 \%$ & $-10 \%$ \\
\hline & Strongl & $9 \%$ & $26 \%$ & $16 \%$ & $11 \%$ & $32 \%$ & $21 \%$ & $11 \%$ & $28 \%$ & $17 \%$ & $31 \%$ & $38 \%$ & $7 \%$ \\
\hline & Agree and Strongly Agree & $51 \%$ & $73 \%$ & $22 \%$ & $75 \%$ & $89 \%$ & $14 \%$ & $82 \%$ & $88 \%$ & $6 \%$ & $88 \%$ & $85 \%$ & $-3 \%$ \\
\hline \multirow{5}{*}{4} & \multicolumn{13}{|c|}{ Fluid mechanics research examples should be presented in the classroom. } \\
\hline & Mean Rating & NA & NA & NA & 3.89 & 4.21 & $8 \% *$ & 4.04 & 4.32 & $6 \% *$ & 4.47 & 4.54 & $1 \%$ \\
\hline & Agree & NA & NA & NA & $68 \%$ & $58 \%$ & $-10 \%$ & $60 \%$ & $56 \%$ & $-4 \%$ & $41 \%$ & $31 \%$ & $-10 \%$ \\
\hline & Strongly Agree & & NA & NA & $11 \%$ & $32 \%$ & $21 \%$ & $25 \%$ & $40 \%$ & $15 \%$ & $53 \%$ & $62 \%$ & $9 \%$ \\
\hline & Agree and Strongly Agree & NA & NA & NA & $79 \%$ & $89 \%$ & $11 \%$ & $85 \%$ & $95 \%$ & $11 \%$ & $94 \%$ & $92 \%$ & $-2 \%$ \\
\hline \multirow{5}{*}{5} & \multicolumn{13}{|c|}{ I am interested in getting involved with fluid mechanics related undergraduate research. } \\
\hline & Mean Ra & $\mathbf{N}$ & $\mathbf{N A}$ & NA & 3.00 & 3.42 & $12 \% *$ & 3.18 & 3.20 & $1 \%$ & NA & NA & NA \\
\hline & Agree & NA & NA & NA & $21 \%$ & $37 \%$ & $15 \%$ & $26 \%$ & $23 \%$ & $-3 \%$ & NA & NA & NA \\
\hline & Strongl & $\mathbf{N}$ & $\mathbf{N A}$ & NA & $0 \%$ & $11 \%$ & $11 \%$ & $13 \%$ & $11 \%$ & $-2 \%$ & NA & NA & NA \\
\hline & Agree and Strongly Agree & NA & NA & NA & $21 \%$ & $47 \%$ & $26 \%$ & $39 \%$ & $34 \%$ & $-5 \%$ & NA & NA & NA \\
\hline
\end{tabular}

\section{Students' Overall Assessment}

Another important aspect of the assessment instruments pertained to how valuable the students rated this research transfer experience/intervention. Figure $\mathbf{4}$ shows the results of four questions, which were based on a scale of 1 to 5 (strongly disagree to strongly agree), for all the groups. Overall, students highly rated the experience and gave it a mean rating above 4 . Interestingly, the graduate students ranked it highest (4.42). Students also agreed that the experience was valuable for teaching them about engineering as an interdisciplinary profession and also about the diversity of the profession. This is consistent with previous results presented from Table 2. 


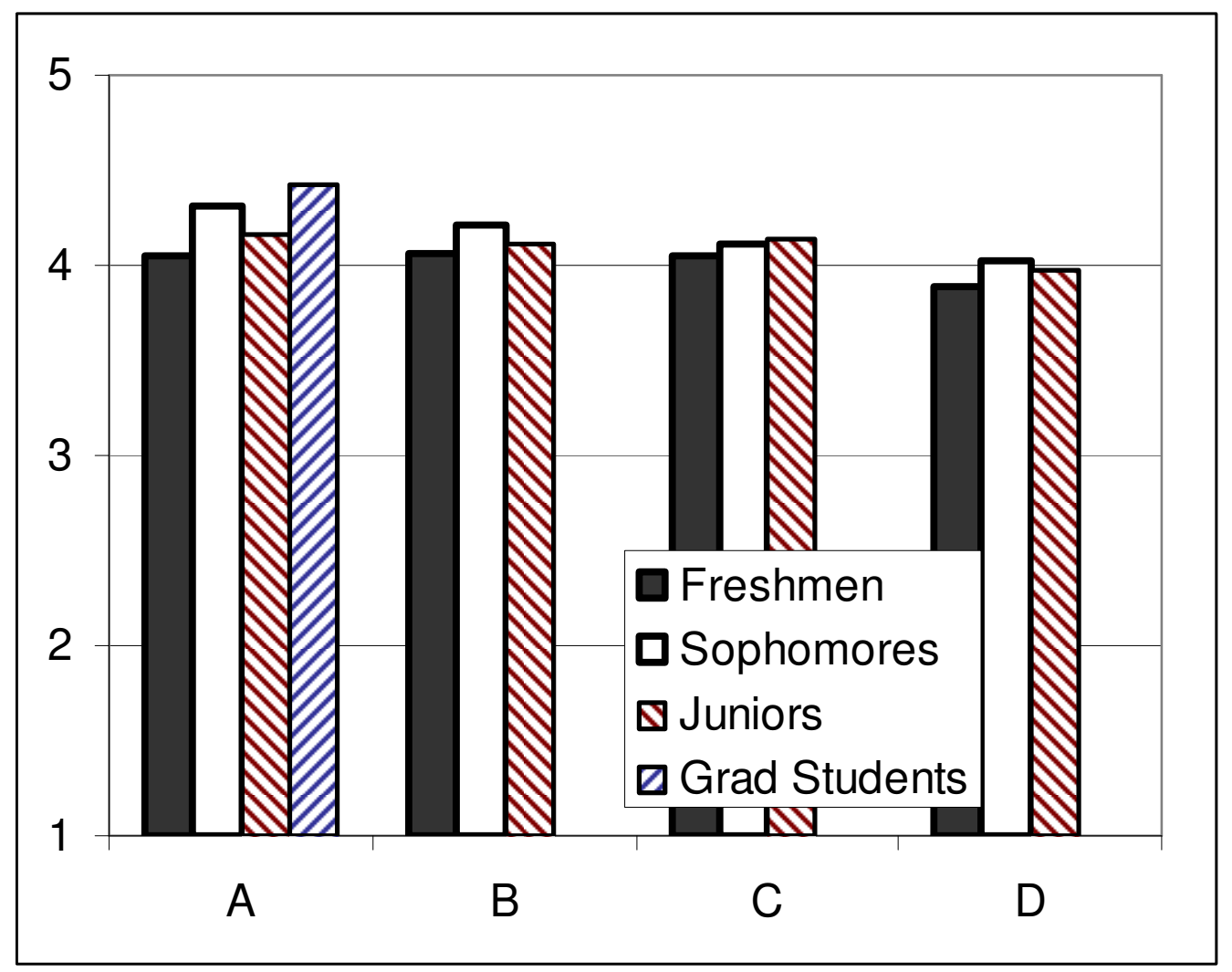

Figure 4: Student survey questions and results pertaining to the value of the intervention showing the mean rating on a scale of 1 to 5 of the answers to each question.

\section{Survey Questions}

A. Overall, this presentation was a valuable learning experience.

B. The experience was valuable for learning about engineering as an interdisciplinary profession.

C. The presentation was valuable for learning about the diversity of engineering.

D. The presentation/experience gave me a clear picture of the relevance of engineering research.

\section{Conclusions}

This paper has presented and evaluated the transfer for state-of-the-art fluid mechanics and biofluids research into the engineering education of students from the freshman level to the graduate level. For the undergraduates, the goal was to introduce them to various research areas as a means of illustrating the diversity of research and the overlap of fundamental engineering concepts. Overall, the findings illustrate that the effort has much promise as a model for transferring engineering research to all academic levels.

To better educate the students that engineers transcend the boundaries of a singlediscipline, several examples were given to illustrate the multidisciplinarity of most engineering projects in industry and academic settings. Assessment results revealed that exposing students to research examples, the multidisciplinary and interdisciplinary nature of engineering can be taught. There was also an increased recognition of 15-20 percentage points of the students who 
agreed or strongly agreed that research has a large impact on their daily lives, and an increased interest in undergraduate research among all the undergraduate students.

On average, $90 \%$ of the students (corresponding to a $30 \%$ increase when comparing pre and post results) exposed to this intervention agreed and recognized that fluid mechanics has a large impact on their daily life. This was a significant outcome not only because so many students recognized the impact of fluid mechanics, but also because students at all levels acknowledged this effect. Even the graduate students, most of whom are doing fluid mechanics research at the graduate-level, realized the influence and relevance of fluid mechanics in their lives. The presentation also improved the perception among sophomores and juniors of the need for fluid mechanics instruction in the general engineering curriculum.

One broader impact of this experience is that the students who go through the program will have a better awareness of engineering and the future direction engineering is taking as a profession. This has been supported by our assessments of students that have participated in our presentations. A positive research experience can be critical to a student's decision about graduate education. This effort, which appears to significantly improve students' image of what is engineering, should also deepen their appreciation of research and graduate studies as a career path, thus resulting in more graduate students who may also be more open to working with undergraduates because of the benefits they earned. Hopefully, the increase in interest in undergraduate research will be followed by a corresponding increase among these students of actual involvement in the programs. One thing we would like to investigate further in the future is to track the students who have participated in this program, to see if the short-term improvements in perception of research and fluid mechanics will in fact translate into improved retention at higher academic levels. If proved to be as useful as initial results indicate, the structure of this experience can readily be transferred to other engineering disciplines, academic levels, and types of institutions.

\section{Acknowledgments}

The authors would like to acknowledge Dr. Bevlee Watford, Director of CEED, Dr. Mark Paul, Dr. Mark Cramer, Whitney Edmister, Trey Waller, Brad Matanin, and Matt Stimpson for their support during this effort.

\section{References}

[1] Boyer Commission on Education of Undergraduates in the Research University, Reinventing Undergraduate Education: A Blueprint for America's Research Universities (New York: 1998).

[2] National Science Foundation, New Expectation for Undergraduate Education in Science, Mathematics, Engineering, and Technology (Washington, DC: NSF Directorate for Education and Human Resources, June 1996), pp.ii, 2, 4, 21, 41, 51, 65.

[3] American Association for the Advancement of Science, Project 2061 Update (Washington, DC: AAAS, 20012002).

[4] National Research Council, Evaluating and Improving Undergraduate Teaching in Science, Technology, Engineering, and Mathematics (Washington, DC: National Research Council, 2003), p. 116.

[5] National Academy of Engineering of the National Academies, "Education the Engineer for 2020: Adapting Engineering Education to the New Century,” The National Academies Press: Washington, D.C., 2005. 